\title{
Dynamic effective elasticity of melanoma cells under shear and elongational flow confirms estimation from force spectroscopy
}

\author{
A. M. Jötten ${ }^{1}$, S. Neidinger ${ }^{1}$, J.K. Tietze ${ }^{2}$ J. Welzel ${ }^{3}$, C. Westerhausen ${ }^{1,4,5}$ \\ ${ }^{1}$ Experimental Physics I, Institute of Physics, University of Augsburg, 86159 Augsburg, Germany \\ 2 Clinic and Policlinic for Dermatology and Venerology, University Medical Center Rostock, 18057 Rostock \\ 3 Department of Dermatology, University hospital Augsburg, 86179 Augsburg, Germany \\ 4 Physiology, Institute of Theoretical Medicine, University of Augsburg, 86159 Augsburg, Germany \\ ${ }^{5}$ Center for NanoScience (CeNS), Ludwig-Maximilians-Universität Munich, 80799 Munich, Germany
}

corresponding author: christoph.westerhausen@gmail.com

\section{ABSTRACT}

The detection and enrichment of circulating melanoma cells is a challenge as the cells are very heterogeneous in terms of their biomechanical properties and surface markers. In addition, there is a lack of valid and reliable biomarkers that predict progress and therapeutic response. We here analyzed the elasticity of A375 melanoma cells applying force spectroscopy and a microfluidic method. To identify and eventually separate circulating tumor cells, it is crucial to precisely know their physical properties. First, we used standard AFM force spectroscopy, where the elasticity of the cells is calculated from indentation with a pyramidal tip. To extend the limits of measurement with a tip, we then used cantilevers without a tip to press on the cells over a large area. The resulting Young's moduli are slightly lower and vary less without tip presumably because of the inhomogeneity of the cells. Finally, we implemented our microfluidic method. We measured single cell elasticity by analyzing its deformation in highspeed micrographs while passing a stenosis. Combining the force field and the change in shape provides the basis for a stress strain diagram. The results from microfluidic deformation analysis were in accordance with the results from force spectroscopy. The microfluidic method provides advantages over conventional methods, since it is less invasive and less likely to harm the cell during the measurement, and the whole cell is measured as one entity without contact to a stiff substrate, while force spectroscopy is limited to the contact area of the tip, and in some cases dependent of the cell substrate interaction. Consequently, microfluidic deformation analysis allows to predict the overall elastic behavior of the whole inhomogeneous cell in three-dimensional force fields. This method may contribute to improve the detection of circulation melanoma cells in the clinical practice. 


\section{INTRODUCTION}

During the last decade circulation tumor cells gained increasingly attention as a prognostic biomarker[1]. They may be detected at any time of the disease and have been associated to disease prognosis, prediction of response to treatment, early detection of treatment resistance or simply to gain tumor cells for analysis, when a biopsy cannot be taken[2], [3]. Challenges are for once the low concentration of CTCS, and their physical similarity to leucocytes. In malignant melanoma in particular, there is great heterogeneity in the circulating melanoma cells with regard to their surface markers and the physical cell properties such as size and plasticity[4], which makes enrichment and sorting challenging. Pathological changes such as tumor formation and metastasis often become apparent through altered mechanical properties of the cells [5]. The deformability plays a crucial role in extravasation of both leukocytes and melanoma cells [6]. To use these properties to optimize e.g. a mechanical filter system, it is of vital importance to determine small differences in elasticity and size between different cell types. The A375 ( ATCC $^{\circledR}$ CRL-1619 ${ }^{\text {TM }}$ ) cell line is a human melanoma cell line initiated through explant culture of a solid tumor from a 54-year-old female and has been used e.g. to obtain paracrine factors for prolonged culture of mesenchymal stromal cells [7]. On that basis, we study the elasticity of A375 melanoma cells using force spectroscopy and microfluidic deformation analysis.

Common methods of determining elasticity are particle-tracking microrheology, optical stretching, micropipette aspiration and microindentation [5], [8], [9]. In particle-tracking microrheology, fluorescent particles are injected into cells to visualize thermal vibrations [10]. How the particles themselves might affect the measured mechanical properties is not clear [5]. Optical stretching combines a dual laser beam and a microfluidic system. The cells can be caught in an optical trap and stretched in a well-defined manner. Here the elasticity is calculated from the changes in refractive index [8]. The concept of micropipette aspiration is to apply negative pressure to a pipette tip that is in contact with the cell surface [11]. The elasticity calculated from the deformation of the aspired piece of membrane predominantly describes the properties of the membrane, and not of the entire cell body [5]. Force spectroscopy using an atomic force microscope (AFM) or, more general, microindentation is a method to punch an indenter with well-defined geometry into a cell. The resulting forceindentation curves allow for determination of the Young's moduli of cells by fitting them, e.g. to the Hertz model [5]. Depending on the method, the level of applied mechanical stress, the rate of deformation, the geometry of the probe, the location probed in the cell, and the extracellular microenvironment, the results can vary by up to a factor of 1000 [8].

Hochmuth et al. use micropipette aspiration to distinguish cell types that behave like a fluid drop from such that behave like a solid. Applying the negative pressure to the cell surface, the length of the aspirated membrane section increases linearly with increasing absolute pressure. Once aspirated, a fluid-like cell overcomes a critical pressure, above which it will slide into the pipette without resistance. For a solid-like cell, the linear relation of pressure and aspirated length is sustained. They find neutrophils to behave fluid-drop like with a surface tension of about $30 \mathrm{pN} / \mu \mathrm{m}$ and a viscosity on the order of $100 \mathrm{~Pa} \mathrm{~s}$, while chondrocytes and endothelial cells are 100 times stiffer and behave solid-like with an elastic modulus of the order of $0.5 \mathrm{kPa}$ [12]. 
Hu et al. developed a microcytometer to calculate the cell's elasticity from its deformation when squeezing into a microfluidic constriction. They distinguish between small and large deformation and find cancerous cells with $E \approx 2 \mathrm{kPa}$ significantly softer under small deformation than a normal breast epithelial cell line with $E \approx 3 \mathrm{kPa}$. Under large deformation, however, when also the cell nucleus is deformed, the Young's modulus rises to $E \approx 4 \mathrm{kPa}$ and the difference between the cell lines vanishes [13].

Loizeau et al. measure metastatic WM239 melanoma cells by force spectroscopy with a spherical tip and find low values of $E \approx 0.13 \mathrm{kPa}$. Moreover, Kuznetsova et al. present elastic moduli of various cell lines reviewing the AFM force spectroscopy results of several groups. They report leukocytes to be significantly softer $E \approx 0.1 \mathrm{kPa}$ than any other cell type. Endothelial cells, osteoblasts, astrocytes and fibroblasts all range in between $E \approx 5-10 \mathrm{kPa}$, erythrocytes and muscle cells are harder $E \approx 15 \mathrm{kPa}$. The highest reported Young's Modulus exhibit cardiocytes with $E \approx 100 \mathrm{kPa}$ [14].

There is a lack of a comprehensive view of melanoma cell elasticity, particularly one that describes the elasticity at the whole cell level. Here we apply two methods to determine this for the melanoma model cell line A375 to fill this gap. The first approach is AFM force spectroscopy, where we step away from the usual cantilever with tip. A pointy tip carries the risk of puncturing and damaging the cell [15], while a large, spherical tip may catch more of the substrate [16]. Abandoning the tip and pressing on the cell with the flat side of the $20 \mu \mathrm{m}$ wide cantilever, we measure not only local elastic properties, but rather the elasticity of the whole cell as a homogeneous object.

However, even for small indentations an indirect influence of the substrate to the elasticity of the cell is possible. Thus, especially for cells, which in vivo either grow in a soft environment or circulate in the blood flow, it might be more appropriate and advantageous to use a method, which studies the cell as one whole object in a fluid instead of punctuating it. Thus, we additionally employ a microfluidic method of that kind. 


\section{METHODS}

\section{Cell culture}

The $\mathrm{A} 375$ cells $\left(\mathrm{ATCC}^{\circledR} \mathrm{CRL}-1619^{\mathrm{TM}}\right.$ ) studied here are human malignant melanoma cells. They were cultivated under standard conditions of $37^{\circ} \mathrm{C}$ and $5 \%$ CO2 in DMEM (30-2002, ATCC, Virginia, United States) containing 10\% FBS (Bio\&Sell) and 1\% Penicillin-Streptomycin (SigmaAldrich).

\section{Force Spectroscopy}

Force spectroscopy on adherent A375 melanoma cells was performed with a NanoWizard BioAFM (JPK instruments AG, Berlin, Germany) on PLL-coated glass (coating with Poly-L-Lysine, $0.1 \% \mathrm{w} / \mathrm{v}$, in water, Thimerosal, $0.01 \%$, added as preservative, SIGMA ALDRICH CHEMIE GMBH, Germany), diluted in phosphate buffered saline (4:996 PLL:PBS) and incubated for 1 hour at room temperature) in DMEM suspension. The cell was approached at $1 \mu \mathrm{m} / \mathrm{s}$ until reaching a setpoint of $2 \mathrm{nN}$, followed by retraction after a pause of $1 \mathrm{~s}$. The first micrometer of indentation is input to extract the Young's modulus from the approach force distance curves. Figure 1 shows an example of one measurement including the approach and retract curves. In force spectroscopy, usually several hundred of such scans are performed for one measurement. To avoid damages by the frequent indentation, the number of scans is reduced to typically 10 measurement curves per cell for this work.

For the measurements AFM with tip a pyramid-shaped tip of the type MLCT-BIO (Bruker company, Massachusetts, United States) with an edge length of about $3 \mu \mathrm{m}$, tip radius $20 \mathrm{~nm}$, an angle of $\alpha=35^{\circ}$, and a cantilever stiffness of $30 \mathrm{mN} / \mathrm{m}$ is used. AFM without tip implies, that cantilevers with $20 \mu \mathrm{m}$ width without a tip are used (MLCT-010-D, Bruker Company, Massachusetts, United States). To adjust the model underlying the fit function, we approximate the geometry by a spherical shape with radius $\mathrm{R}=10 \mu \mathrm{m}$.

\section{Microfluidic Method Microchannel design and measurement setup}

The microchannel consisted of a polydimethylsiloxane (PDMS) single layer fabricated by standard soft lithography [17], which was bonded on a glass slide by plasma activation. Generally, all channels were $7 \mathrm{~mm}$ long, $27 \mu \mathrm{m}$ in depth, and $50 \mu \mathrm{m}$ in width. The central part of interest is comprised of a single stenosis, see Figure $4 \mathrm{~A}$, in which the channel width was reduced from $50 \mu \mathrm{m}$ to $5 \mu \mathrm{m}$ or $7 \mu \mathrm{m}$. The setup consisted of the microchannel, a pipet tip serving as the cell suspension reservoir, and a syringe pump (Harvard PHD2000, Harvard Apparatus). A Hamilton Gastight Syringe (Hamilton Bonaduz AG) containing phosphate buffered saline (PBS, $\mathrm{pH}=7.4$ ) was connected to the microchannel via a polyfluoroethylene (PTFE) tube. The pump was operated in reverse mode to provide a controlled flow by drawing the suspension from the reservoir through the microfluidic channel at a flowrate of $Q=75 \mu \mathrm{l} / \mathrm{h}$. We observed the cells using the 20x objective of an inverted microscope (Zeiss Axiovert200) equipped with a high-speed video camera (FASTCAM Mini UX50 type 160K-M-16G, Photron). The videos were recorded with the software Photron Fastcam Viewer PFV from Photron at 2000 frames per second, $1280 \times 120$ pixel resolution, $1 / 40000 \mathrm{~s}$ exposure resulting in a maximum total of 74531 frames or $37 \mathrm{~s}$ in real time covering an area of $704 \times 66 \mu \mathrm{m}^{2}$. For analysis, we adapted our customized software SURF, introduced earlier [18], [19]. 
RESULTS

Elasticity of melanoma cells - force spectroscopy

A

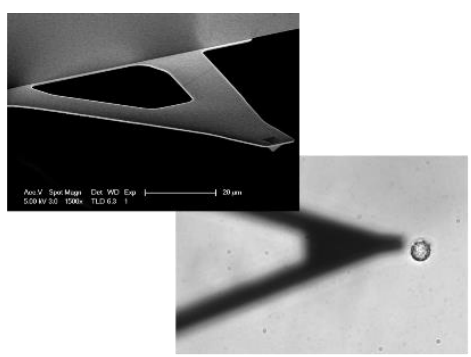

C

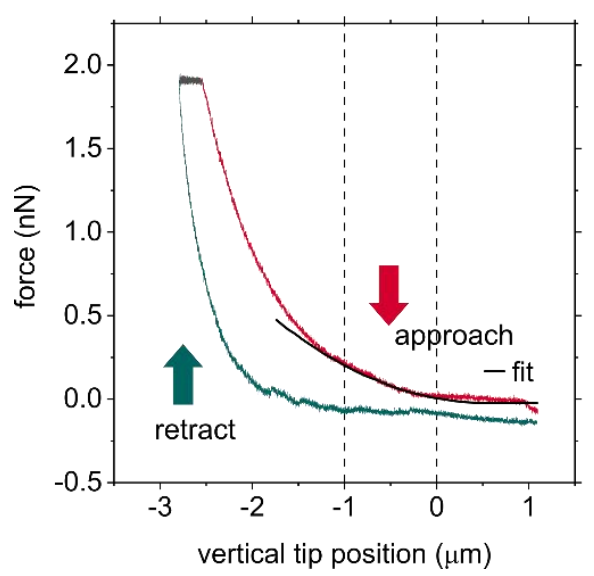

B
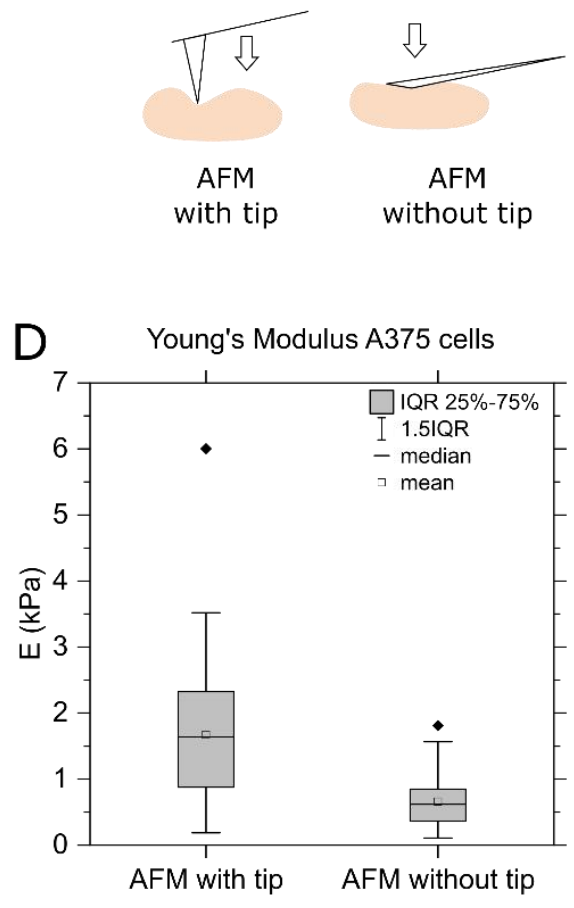

Figure 1 A) Cantilever with pyramidal tip type MLCT-BIO and micrograph with cantilever without tip approaching an adherent A375 cell. B) Principle AFM with and without tip. C) Force-distance curve of an exemplary measurement with cantilever stiffness $k=0.03 \mathrm{~N} / \mathrm{m}$, force setpoint $f=2 \mathrm{nN}$, approaching speed $v=1 \mu \mathrm{m} / \mathrm{s}$ and approaching distance $d=5 \mu \mathrm{m}$. The pause between approach and retract is $1 \mathrm{~s}$ at constant force. D) Comparison of AFM force spectroscopy results of the Young's modulus of A375 cells using a cantilever with tip and one without tip. The boxplot summarizes the results on $\sim 50$ cells with $\sim 10$ force curves per cell of AFM measurements with and without tip. The cantilever without tip is $20 \mu \mathrm{m}$ wide at the front, and the tip radius of the approximately $3 \mu \mathrm{m}$ long tips used here is $20 \mathrm{~nm}$.

Fig. 1A shows an adherent A375 cell on a slide coated with PLL and the cantilever with and without tip. As illustrated in Figure 1B, the Young's modulus of these cells was determined using conventional cantilevers with a pyramidal tip and with a tipless cantilever. Typical indentation curves are shown in Figure 1C. By fitting the indentation curves in the indentation $\delta$ range of $0 \mu \mathrm{m}<\delta<1 \mu \mathrm{m}$ and applying a Hertz-model, the Young's modulus $E$ was extracted from each curve. For both the AFM measurements with and without tip, typically the first ten indentation curves of each cell were analyzed. More extensive tests with tip show that for $n=$ 100 repetitions the cell appears slightly harder, and from $n=200$ on significantly harder than at the beginning (see SI Figure S1).

For the two different measuring methods AFM with tip and AFM without tip, the values for the elasticity of the A375 cells differ as shown in Figure 1D. Without tip we found the elastic modulus to be $\mathrm{E}=660 \mathrm{~Pa} \pm 371 \mathrm{~Pa}$. With tip, the elastic modulus is higher with $\mathrm{E}=1671 \mathrm{~Pa} \pm$ $1111 \mathrm{~Pa}$, and the error bar is larger. For each method, with and without tip, 50 cells were measured over three measurement days, involving 10 force curves for each cell. The sample ICC value [20] comparing the range of the results on one day to the variation between the measurement days is close to zero in the without tip case $\left(I C C_{\text {day, no tip }}=-0.08\right)$, and with tip we find a low ICC of ICC day, tip=0.2. Comparing the scattering of the values within one cell to the differences between cells, however, results in high ICC values $\left(I C C_{\text {cell, no tip }}=0.84, I C C_{\text {cell, tip }}=0.66\right)$. 
Concluding, the values correlate strongly within a cell, and therefore only mean values over each cell are summarized in the box plot here. The low ICC day values justify to treat each cell as an independent measurement. The lower correlation and larger error bars for the results with tip might arise for two reasons: If the cell is penetrated, higher values may occur because the underlying harder substrate contributes to the effective elasticity[21]. In addition, the inhomogeneity of the cell has a greater influence on the result with a smaller contact area.

To quantify and describe the alluded inhomogeneity of the cells, we move on to scanning single cells systematically with an AFM tip choosing equidistant measurement positions with $x$ - and $y$-spacing of $1.5 \mu \mathrm{m}$. Figure $2 \mathrm{~A}$ shows an exemplary map of an A375 cell. The central part, where the nucleus is located, compare Fig. $2 \mathrm{C}$, is roughly 10 times harder than the outer areas, compare Fig. 2B.
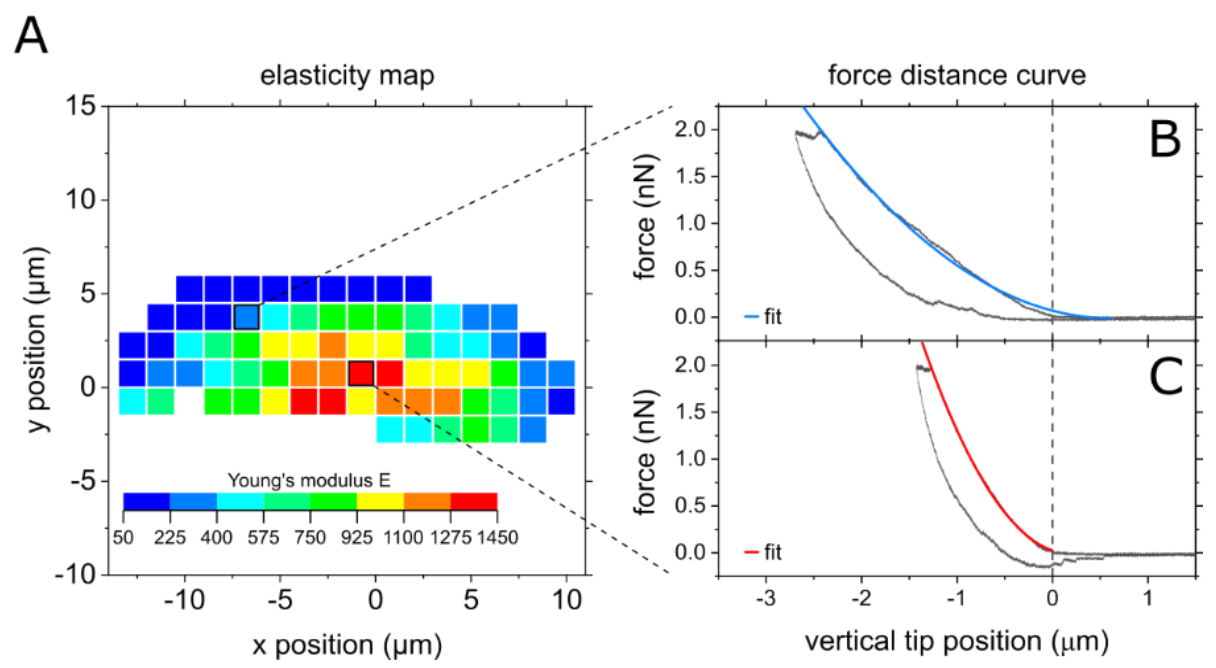

Figure 2 AFM force spectroscopy mapping of a cell using a cantilever with tip. A) Map of half a cell, measurement started at the center of the cell. B) typical force distance curve from the softer outer area, C) typical force distance curve from the central, stiffer part.

The literature here offers a broad range of reported results. In summary, the morphology and elasticity landscape appear to depend strongly on the cell type. Hayashi et al. compare human cervical cancer cells and normal cervical cells and find the cancerous cells to be softer, but comparing central and peripheral elasticity, cancerous cells appear homogenous while the normal cells, like the A375 cell shown here, had a stiffer central body [22]. Galajda et al. image confluent human brain endothelial cells and find, contrary to our results, the cell body to be softer than the outer areas [23].

To investigate further, how sensitive the results depend on the measurement method, we continue with varying the loading rate in force spectroscopy measurements without tip. As results differ strongly from cell to cell, we here normalize the Young's modulus at different loading rates to a reference Young's modulus determined at a loading rate of $a=1.0 \mu \mathrm{m} / \mathrm{s}$ to be able to compare different cells, as shown in Fig. 3. In Figure 3 we show $E$ as function

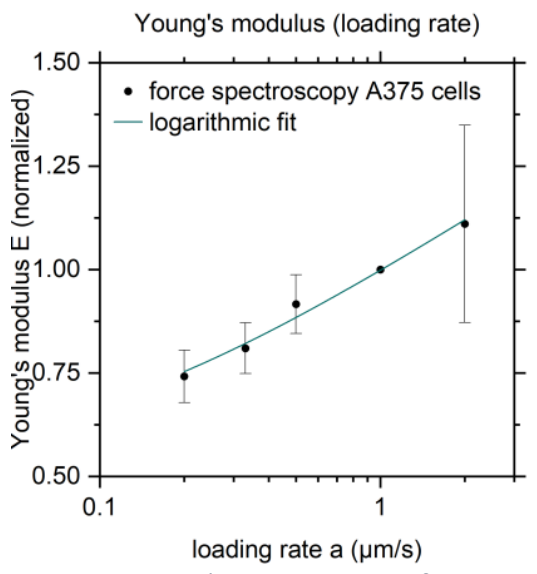

Figure 3 Young' modulus $E$ as a function of the loading rate normalized to the Young's modulus from reference measurements with a loading rate $a=1.0 \mu \mathrm{m} / \mathrm{s}$. The logarithmic fit function is $E=0.98+0.19 \ln (a+0.1)$. 
of the cantilever's approaching velocity, as this was the modified parameter. The actual loading rate in the unit $\mathrm{N} / \mathrm{s}$ slightly differs from cell to cell, due to the variation in elasticity between the cells. The Young's modulus $E$ as a function of the logarithmic loading rate $a$ shows a linear relation. Lowering the loading rate by a factor of five entails a decrease in Young's modulus of $25 \%$. Doubling the loading rate however raises the Young's modulus by $10 \%$.

Li et al. study the elastic modulus of cancerous and benign human breast epithelial cells by AFM indenting with spherical tips and find hardening with increased loading rate as well, but the effect is stronger than in our experiments. The authors of that study report, that benign cells had 1.4-1.8 times higher Young's moduli than malignant cells, and the hardening effect is more pronounced [24]. Ren et al. vary the loading rate even over three orders of magnitude (loading frequency $f=0.2-100 \mathrm{~Hz}$ ) to measure the elasticity of prostate cancer cells. The resulting Young's moduli range from $2 \mathrm{kPa}$ at $\mathrm{f}=0.2 \mathrm{~Hz}$ to $10 \mathrm{kPa}$ at $\mathrm{f}=100 \mathrm{~Hz}$ for untreated cells. They state, that the power law relation between Young's modulus and frequency they find is characteristic for live human cells [25]. Moreover, Pogoda et al. study fibroblasts and A375 cells using an AFM with pyramidal tip for force spectroscopy. They average the measurements on a 12×12 point grid on each cell and find lower Young's moduli upon deeper indentation. For indentation depths $>800 \mathrm{~nm}$ the value is stable at $\mathrm{E}=760 \mathrm{~Pa} \pm 370 \mathrm{~Pa}$ [26], which agrees with our results.

However, even for small indentations an indirect influence of the substrate to the elasticity of the cell is possible. Thus, especially for cells, which in vivo either grow in a soft environment or circulate in the blood flow, it might be more appropriate and advantageous to use a method, which studies the cell as one whole object in a fluid instead of punctuating it. Thus, we additionally employ a microfluidic method of that kind. 


\section{Elasticity of melanoma cells - microfluidic method}

Finally, the deformation of $A 375$ cells is analyzed during passage of a microfluidic channel with a diameter of $27 \times 50 \mu \mathrm{m}^{2}$ and a stenosis of $27 \times 5 \mu \mathrm{m}^{2}$. At a flow rate of $\sim 75 \mu \mathrm{l} / \mathrm{h}$ high speed micrographs with $2000 \mathrm{fps}$ and 1/40000 s exposure are recorded. Exemplary micrographs are shown in Figure 4A. The cells are traced from frame to frame using the customized MATALB script SURF [19].

We calculate the elastic modulus from the stress-strain-diagram. The strain $\varepsilon$ is directly extracted from the images, where an ellipse approximates the cells' outlines. The values for major and minor axis are plotted as a function of $x$-position in the channel for all traced cells and fitted by an exponential function, to describe phenomenologically the deformation by one representative, continuous function combining the data of $\sim 2500$ cells, compare Figure $4 \mathrm{C}$. The strain $\varepsilon$ is the relative change from the initial diameter of a round cell $d=2 r_{0}$ to the elongated length $L$, which is the major axis of the ellipse, as shown in Figure 4A.

$$
\varepsilon=\frac{L-2 r_{0}}{2 r_{0}}
$$

To calculate the strain, which is the force per area, we follow Dudani et al. and Armistead et al. and transfer their approach to calculate the forces in a "hydropipette", a cross channel, where the cells are compressed by confluent streams. Assuming that the narrowing walls of a stenosis are comparable to Dudani et al.'s "hydropipetting", we find the following expression for the total force $F_{S t}$ on the flowing object in a microfluidic channel assuming a compressive component $F_{C}$ and a shear force component $F_{S}$ including the parameters fluid density $\rho$, fluid viscosity $\mu$, velocity v, drag coefficient $C_{W}$, cell cross section $A_{S}$ and cell radius $r$ [27], [28].

$$
F_{S t}=F_{C}+F_{S}=\frac{1}{2} \rho v^{2} C_{W} A_{S}+2 \pi r \mu v
$$

As the force varies significantly over the extent of a cell, at each position in the channel, the strain is calculated as weighted average of the force per area in steps of one pixel from cell rear to cell front.

In summary, the stress acting on the cells is calculated from the velocity (Figure 4 4C), the cell size, and the resulting drag force and shear force, to plot the stress as a function of the strain. For linear elastic materials, the Young's modulus is the slope of the stress-strain-diagram. Such a diagram is shown in Figure 4D, it summarizes all 2500 cell trajectories of one measurement. Figure 4E shows the resulting Young's moduli for six independent measurements ranging from $E=300 \mathrm{~Pa}$ to $\mathrm{E}=1000 \mathrm{~Pa}$.

For comparison, we examine the work of Otto et al. on the mechanical characterization of cells using flow cytometry [29], [30]. Adapting their model and using the deformation and size of the cells studied here as input, we can predict the Young's modulus to be in the range of $E=500$ $800 \mathrm{~Pa}$, which is in line with our results. 
A

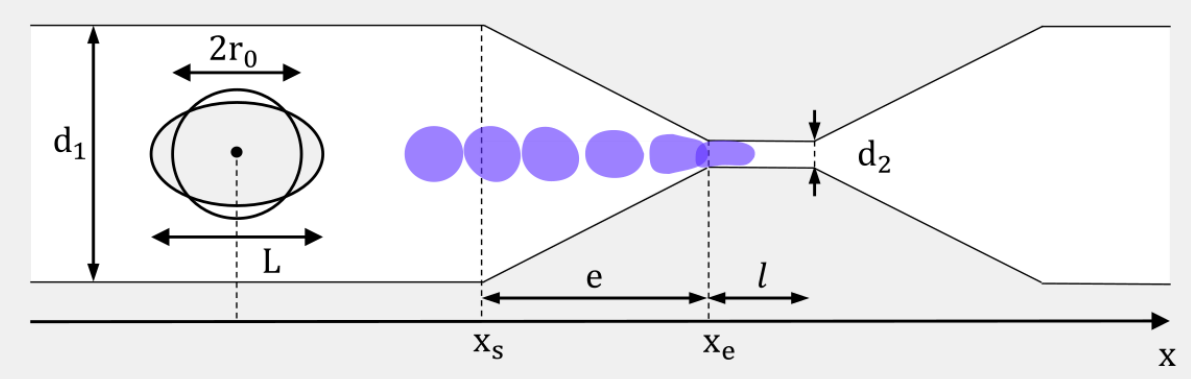

B

C
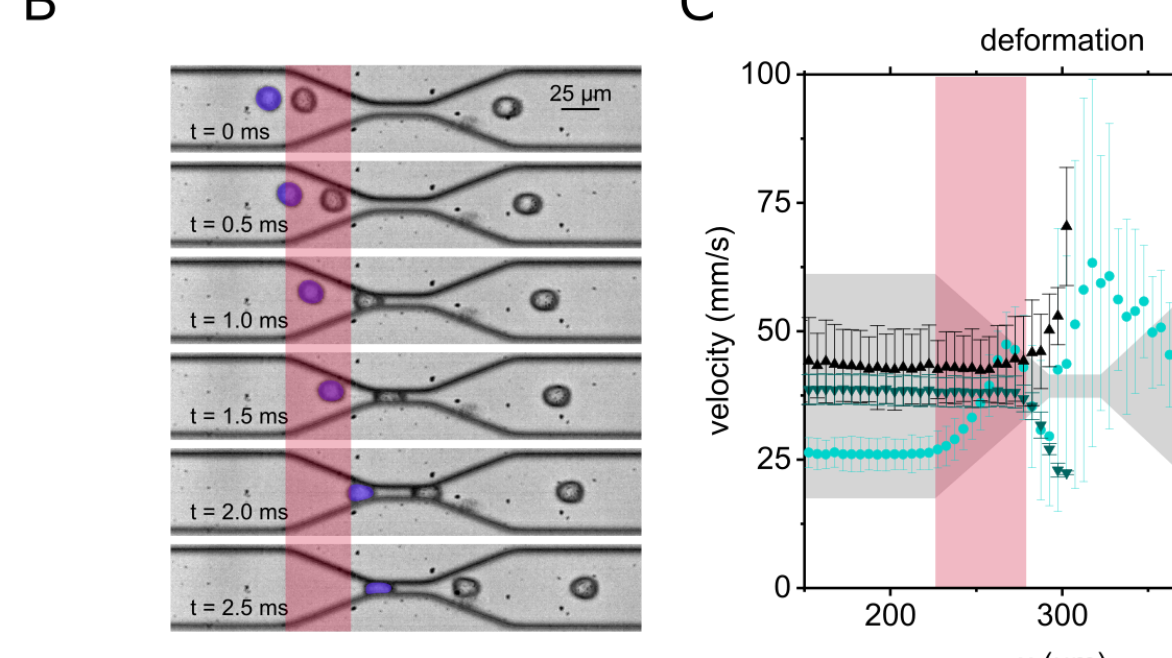

D

stress strain diagram

$\mathrm{E}$

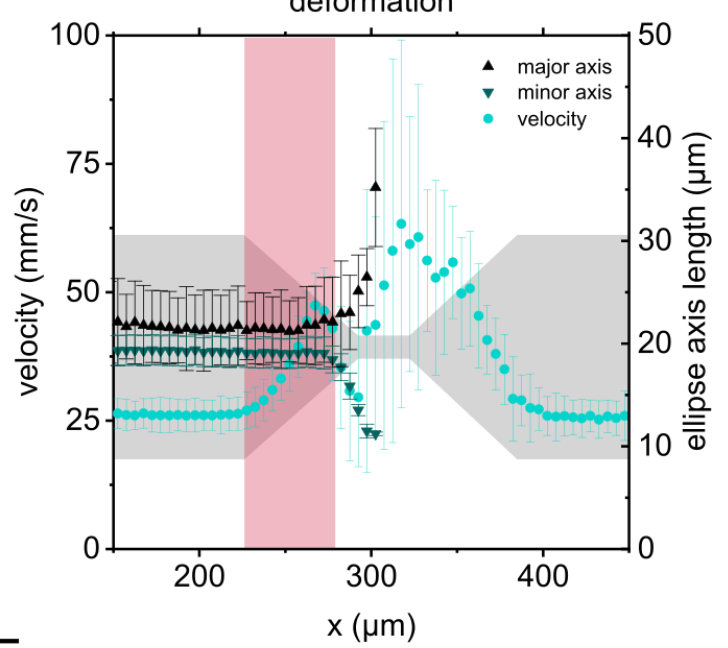

A375 cell deformation analysis
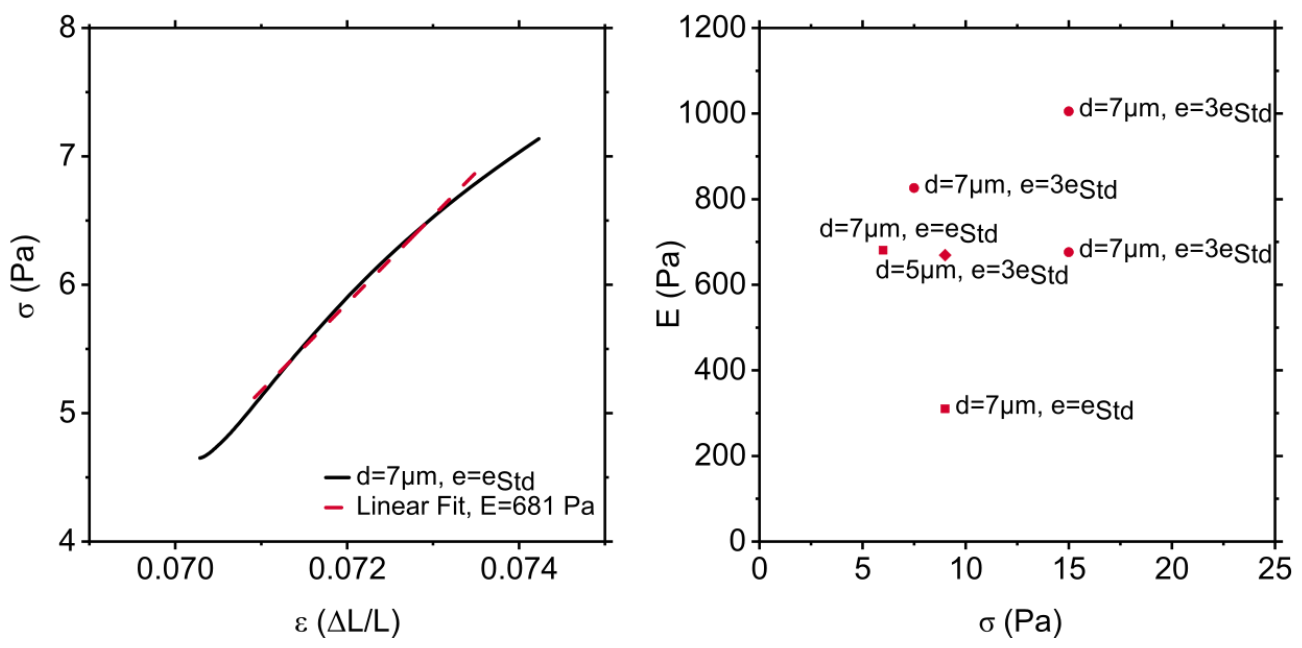

Figure 4 A) Design of the microfluidic stenosis narrowing from diameter $d_{1}$ to diameter $d_{2}$ over the elongational length e. The cell deforms from a round shape with its original diameter $d=2 r_{0}$ to an ellipse with major axis $L$. B) Exemplary micrographs of A375 cells passing a standard elongation $d=7 \mu \mathrm{m}$ stenosis. The area for the deformation analysis highlighted in red. C) Velocity, major and minor axes from one measurement. Mean and standard deviation from 2300 cell trajectories. D) Stress strain diagram and linear fit to determine the Young's modulus of one set of A375 cells. E) Resulting Young's moduli as function of applied stress $\sigma$ from six independent measurements as shown in $D$, representing about 2500 cells each. 


\section{Limitations of the method}

While cells are not harmed punctually as in force spectroscopy when using the microfluidic method, there are indications that the cells do not run through the measurement completely unaffected. Figure 5 shows the deformation of the cells as a function of the x-position. The deformation is measured by approximating the cell's outline by an ellipse and evaluating its eccentricity $\varepsilon$.

Eccentricity designates the ratio of the distance between the foci of the ellipse and its major axis length. The value ranges between 0 and 1 , where the degenerate cases are 0 , a circle and 1 , a line. Before the stenosis, the cells are already slightly elongated with $\varepsilon=0.4$. When passing the stenosis, the cell is deformed and lengthened, and the eccentricity rises to almost 1 , because the stenosis is much narrower than the cell's diameter. After the stenosis, the cells relax, but not fully. The cells remain elongated with $\varepsilon=0.6$ when leaving the camera's field of view. Comparing the ellipses as depicted in Fig. 5 for $\varepsilon=0.4$ and $\varepsilon=0.6$, it should be noted that the difference is measurably large, but not particularly significant. Following the flow field, the cells deform and recover faster in a shorter stenosis with length $e=55 \mu \mathrm{m}$ than passing a longer stenosis with length $e=165 \mu \mathrm{m}$. The more abrupt deformation seems to allow for better recovery. Whether the cells will return to their original shape after a longer period of time cannot be concluded from this data. Additionally, occasional

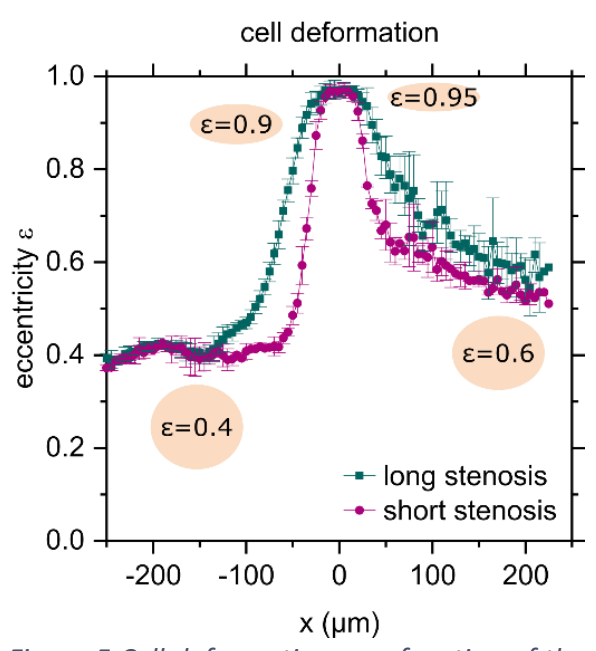

Figure 5 Cell deformation as a function of the $x$-position, the center of the stenosis is at $x=0$. The shape of the cell is approximated by an ellipse. The eccentricity $\varepsilon$ of an ellipse is the distance between the foci divided by the major axis length. The labelled ellipses illustrate the values. "Long" and "short" stenosis refers to stenoses with length $e=$ $165 \mu \mathrm{m}$ and $e=55 \mu \mathrm{m}$, respectively. events of cell rupture or budding were witnessed, when a large cell reached the widening part of the stenosis. Concluding, the microfluidic method comes with limitations. If the cells are particularly sensitive or if the focus lies on the cells' viability after the measurement, those permanent damages have to be taken into account and the design of the stenosis may need to be adapted e.g. by widening the narrowest part. In general, however, the advantages outweigh the limitations, as a large number of cells can be analyzed in one measurement, the analysis is automatic, and the measured quantity does not describe selective points, but actually the bulk elasticity of an entire cell. 


\section{DISCUSSION}

\section{Comparison of measurement techniques}

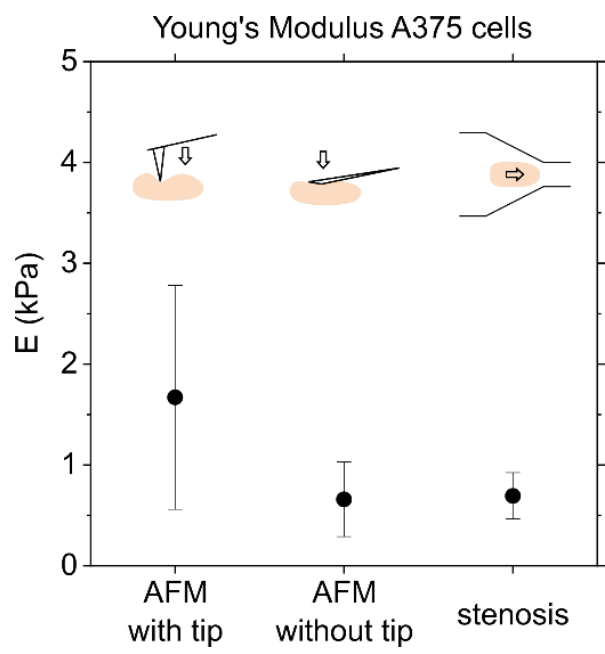

Figure 6 Comparison of AFM and microfluidic deformation analysis of the Young's modulus of A375 cells. AFM: The result of AFM measurements with and without tip is the mean and standard deviation from measurements on 5 - 15 cells with $\sim 10$ force curves per cell. Stenosis: Elasticity was also determined based on deformation under flow during passage of a stenosis. The value here summarizes the six values from Fig. 3, each of which relies on trajectories from approximately 2500 cells.

Figure 6 shows, that different measurement methods result in different values and different measuring uncertainties of the Young's modulus. Under the label stenosis, the results from the microfluidic measurements in Figure 4E are summarized. These result in a value of $\mathrm{E}=695 \mathrm{~Pa}$ $\pm 229 \mathrm{~Pa}$. Comparing AFM with tip ( $\mathrm{E}=1671 \mathrm{~Pa} \pm 1111 \mathrm{~Pa})$, AFM without tip ( $=660 \mathrm{~Pa} \pm 371$ $\mathrm{Pa})$ and stenosis or microfluidic deformation analysis $(\mathrm{E}=695 \mathrm{~Pa} \pm 229 \mathrm{~Pa})$, the results from AFM without tip and stenosis are in agreement. Overall, the results of the three measurement methods are in the same range, but not particularly precise. In the following, we consult other studies to evaluate the broad range of our results.

Guillou et al., who study the deformation of cells during passage of a channel crossing, publish shear moduli of 3T3 fibroblasts between $\mathrm{G}_{0}(200 \mathrm{~ms})=101 \pm 8 \mathrm{~Pa}$ and $\mathrm{G}_{0}(5 \mathrm{~ms})=590 \pm 5 \mathrm{~Pa}$ for different time constants. For validation, they refer to optical stretcher experiments, which lead to values in the range of $70-100 \mathrm{~Pa}$. Applying their microfluidic method to GBM tumor initiating cells, they find $G_{0}=440 \pm 0.03 \mathrm{~Pa}$, and report AFM results of $\mathrm{G}_{0}=800-900 \mathrm{~Pa}$ [31]. Another set of different cell lines and methods is provided by Chen et al., who also developed a microfluidic method, which is based on measuring the changes in pressure while a cell passes a stenosis. The results for K562 leukemia cells are $64 \mathrm{~Pa}$ from the microfluidic method, $90 \mathrm{~Pa}$ from micropipette aspiration and 400 Pa measured by AFM. The same three measurement methods do not show the same systematics for endothelial cells: $383 \mathrm{~Pa}$ from the microfluidic method, 100-400 Pa from micropipette aspiration and 700-3000 Pa using AFM [32].

To summarize, the value of the elastic modulus depends strongly on the measurement method. Comparing different cells' elasticity must therefore always rely on one method. Even within one method, the outcome depends on the details. E.g. in force spectroscopy, low indentation depths result in higher and more volatile results, because the actin network close to the cell's surface is rigid and heterogeneous [26]. Between methods, results in the same range are satisfactory. Having access to a microfluidic method that is non-invasive is of great value, because it yields rapid results, captures information about many cells, and allows for reuse of the cells. Therefore, this application may be a very useful tool in the clinical practices in the future. 


\section{CONCLUSION}

In this study, the elasticity of A375 melanoma cells was determined applying different methods. By force spectroscopy using cantilevers with and without tip and force mapping we emphasize the impact of the inhomogeneity of the cells resulting in higher and more widely distributed values using a cantilever with tip. The presented microfluidic method allows for high-throughput and minimal invasion. The resulting Young's moduli coincide with those from force spectroscopy without tip in the range of $E \approx 700 \mathrm{~Pa}$. The method is therefore a powerful addition to the common ones needed to detect pathological changes in cell elasticity as seen in various diseases starting with cancer, Covid-19, or Malaria.

\section{ACKNOWLEDGEMENTS}

The authors thank Achim Wixforth for fruitful discussions and thank the Augsburg Centre for Innovative Technologies (ACIT) for funding. A.M.J. would like to thank the German Academic Scholarship Foundation. C.W. would like to acknowledge funding for the project "Physical and functional interaction mechanisms at cell membranes and vessel walls" by the University of Augsburg. 


\section{LITERATURE}

[1] C. Dive and G. Brady, "SnapShot: Circulating Tumor Cells," Cell, vol. 168, no. 4, pp. 742-742.e1, Feb. 2017.

[2] G. Marsavela, C. A. Aya-Bonilla, M. E. Warkiani, E. S. Gray, and M. Ziman, "Melanoma circulating tumor cells: Benefits and challenges required for clinical application," Cancer Lett., vol. 424, pp. 1-8, Jun. 2018.

[3] E. Kilgour, D. G. Rothwell, G. Brady, and C. Dive, "Liquid Biopsy-Based Biomarkers of Treatment Response and Resistance," Cancer Cell, vol. 37, no. 4, pp. 485-495, Apr. 2020.

[4] K. Gorges et al., "Intra-Patient Heterogeneity of Circulating Tumor Cells and Circulating Tumor DNA in Blood of Melanoma Patients," Cancers (Basel)., vol. 11, no. 11, p. 1685, Oct. 2019.

[5] G. Thomas, N. A. Burnham, T. A. nn. Camesano, and Q. Wen, "Measuring the mechanical properties of living cells using atomic force microscopy," J. Vis. Exp., no. 76, pp. 1-8, 2013.

[6] C. Mondadori, M. Crippa, M. Moretti, C. Candrian, S. Lopa, and C. Arrigoni, "Advanced Microfluidic Models of Cancer and Immune Cell Extravasation: A Systematic Review of the Literature," Front. Bioeng. Biotechnol., vol. 8, no. August, pp. 1-19, 2020.

[7] L. Kucerova, M. Matuskova, K. Hlubinova, V. Altanerova, and C. Altaner, "Tumor cell behaviour modulation by mesenchymal stromal cells," Mol. Cancer, vol. 9, no. 1, p. 129, 2010.

[8] P. H. Wu et al., "A comparison of methods to assess cell mechanical properties," Nat. Methods, vol. 15, no. 7, 2018.

[9] Y. Hao, S. Cheng, Y. Tanaka, Y. Hosokawa, Y. Yalikun, and M. Li, "Mechanical properties of single cells: Measurement methods and applications," Biotechnol. Adv., vol. 45, p. 107648, 2020.

[10] P. H. Wu, C. M. Hale, W. C. Chen, J. S. H. Lee, Y. Tseng, and D. Wirtz, "High-throughput ballistic injection nanorheology to measure cell mechanics," Nat. Protoc., vol. 7, no. 1, pp. 155-170, 2012.

[11] M. J. Oh, F. Kuhr, F. Byfield, and I. Levitan, "Micropipette aspiration of substrateattached cells to estimate cell stiffness," J. Vis. Exp., no. 67, pp. 1-7, 2012.

[12] R. M. Hochmuth, "Micropipette aspiration of living cells," J. Biomech., vol. 33, no. 1, pp. 15-22, 2000.

[13] S. Hu, R. Wang, C. M. Tsang, S. W. Tsao, D. Sun, and R. H. W. Lam, "Revealing elasticity of largely deformed cells flowing along confining microchannels," RSC Adv., vol. 8, no. 2, pp. 1030-1038, 2018.

[14] T. G. Kuznetsova, M. N. Starodubtseva, N. I. Yegorenkov, S. A. Chizhik, and R. I. Zhdanov, "Atomic force microscopy probing of cell elasticity," Micron, vol. 38, no. 8, pp. 824-833, 2007.

[15] L. Streppa et al., "Prestressed cells are prone to cytoskeleton failures under localized shear strain: An experimental demonstration on muscle precursor cells," Sci. Rep., vol. 8, no. 1, pp. 1-16, 2018.

[16] A. Amiri, F. D. Hastert, L. O. Heim, and C. Dietz, "Reliability of cancer cell elasticity in force microscopy," Applied Physics Letters, vol. 116, no. 8. 2020.

[17] Y. Xia and G. M. Whitesides, "Soft Lithography," Annu. Rev. Mater. Sci., vol. 28, no. 1, pp. 153-184, 1998.

[18] A. M. Jötten, "SURF Survival of Rosettes in Flow," 2021. [Online]. Available: https://github.com/anna-joe-0305/SURF. [Accessed: 12-Jul-2021] 
[19] A. M. Jötten, K. Moll, M. Wahlgren, A. Wixforth, and C. Westerhausen, "Blood group and size dependent stability of $P$. falciparum infected red blood cell aggregates in capillaries," Biomicrofluidics, vol. 14, no. 2, p. 024104, Mar. 2020.

[20] D. Liljequist, B. Elfving, and K. S. Roaldsen, Intraclass correlation - A discussion and demonstration of basic features, vol. 14, no. 7. 2019.

[21] R. E. Mahaffy, C. K. Shih, F. C. MacKintosh, and J. Käs, "Scanning Probe-Based Frequency-Dependent Microrheology of Polymer Gels and Biological Cells," Phys. Rev. Lett., vol. 85, no. 4, pp. 880-883, Jul. 2000.

[22] K. Hayashi and M. Iwata, "Stiffness of cancer cells measured with an AFM indentation method," J. Mech. Behav. Biomed. Mater., vol. 49, pp. 105-111, 2015.

[23] P. Galajda, L. Kelemen, and G. A. Végh, "Micro- and nanotechnology for cell biophysics," Acta Biol. Szeged., vol. 59, pp. 303-321, 2015.

[24] Q. S. Li, G. Y. H. Lee, C. N. Ong, and C. T. Lim, "AFM indentation study of breast cancer cells," Biochem. Biophys. Res. Commun., vol. 374, no. 4, pp. 609-613, 2008.

[25] J. Ren, H. Huang, Y. Liu, X. Zheng, and Q. Zou, "An atomic force microscope study revealed two mechanisms in the effect of anticancer drugs on rate-dependent Young's modulus of human prostate cancer cells," PLoS One, vol. 10, no. 5, 2015.

[26] K. Pogoda et al., "Depth-sensing analysis of cytoskeleton organization based on AFM data," Eur. Biophys. J., vol. 41, no. 1, pp. 79-87, 2012.

[27] F. J. Armistead, J. Gala De Pablo, H. Gadêlha, S. A. Peyman, and S. D. Evans, "Cells Under Stress: An Inertial-Shear Microfluidic Determination of Cell Behavior," Biophys. J., vol. 116, no. 6, pp. 1127-1135, 2019.

[28] J. S. Dudani, D. R. Gossett, H. T. K. Tse, and D. Di Carlo, "Pinched-flow hydrodynamic stretching of single-cells," Lab Chip, vol. 13, no. 18, pp. 3728-3734, 2013.

[29] A. Mietke et al., "Extracting Cell Stiffness from Real-Time Deformability Cytometry: Theory and Experiment," Biophys. J., vol. 109, no. 10, pp. 2023-2036, 2015.

[30] M. Mokbel et al., "Numerical Simulation of Real-Time Deformability Cytometry to Extract Cell Mechanical Properties," ACS Biomater. Sci. Eng., vol. 3, no. 11, pp. 29622973, 2017.

[31] L. Guillou et al., "Measuring Cell Viscoelastic Properties Using a Microfluidic Extensional Flow Device," Biophys. J., vol. 111, no. 9, pp. 2039-2050, 2016.

[32] Z. Chen, Y. Zhu, D. Xu, M. M. Alam, L. Shui, and H. Chen, "Cell elasticity measurement using a microfluidic device with real-time pressure feedback," Lab Chip, vol. 20, no.

13, pp. 2343-2353, 2020. 